\title{
Controlling Dengue: Effectiveness of Biological Control and Vaccine in Reducing the Prevalence of Dengue Infection in Endemic Areas
}

\author{
Bryan Paul ${ }^{*}$, Wai Liang Tham² \\ ${ }^{1}$ Faculty of Science, University of Alberta, Edmonton, Canada \\ ${ }^{2}$ Faculty of Medicine, University of British Columbia, Vancouver, Canada \\ Email: "bpaul@ualberta.ca, "bryanpaul.ian@gmail.com
}

Received 4 September 2015; accepted 16 January 2016; published 19 January 2016

Copyright (C) 2016 by authors and Scientific Research Publishing Inc.

This work is licensed under the Creative Commons Attribution International License (CC BY). http://creativecommons.org/licenses/by/4.0/

\section{(c) (i) Open Access}

\begin{abstract}
With the increased prevalence of dengue infection in tropical countries, concerned members of the public are now pressing their local health ministries to act immediately and effectively in managing the rising numbers of reported cases. This includes reviews of the methodologies and the effectiveness of current combative systems to find other possible novel approaches that might yield better results. One of those novel approaches is the integration of a parasite into mosquito vector, manipulating the parasite-host interaction to reduce the transmission of dengue in endemic hotspots. Another alternative is by Sanofi-Pasteur's dengue vaccine that showed over $60.8 \%$ success rate in reducing severe dengue infection in children aged 9 - 16 during its final clinical implementation phase. This report will compare and contrast these two novel ideas to determine which of the approaches are more likely to be effective in the long run. The aspects covered will include the application, effectiveness, functionality, and problems with these approaches. The results could then be utilised by governments or organizations to select precise and effective methods in reducing the prevalence of dengue infections in their countries.
\end{abstract}

\section{Keywords}

Dengue, Pathogenesis, Pathology, Immunology, Biological Control, Vaccine

${ }^{*}$ Corresponding author.

How to cite this paper: Paul, B. and Tham, W.L. (2016) Controlling Dengue: Effectiveness of Biological Control and Vaccine in Reducing the Prevalence of Dengue Infection in Endemic Areas. Health, 8, 64-74.

http://dx.doi.org/10.4236/health.2016.81008 


\section{Introduction}

Dengue, which uses arthropods and other mosquitoes as vectors, is common in tropical and sub-tropical climates, and is often associated with other diseases such as malaria, chikungunya, yellow fever, St. Louis encephalitis, West Nile virus, Japanese encephalitis, and Tick-borne encephalitis [1] [2]. Commonly transmitted by the mosquitoes Aedes aegypti and Aedes albopictus, dengue is considered one of the fastest growing hemorrhagic viruses worldwide [1]-[4]. It is less well-known due to the localisation of the disease, which is uncommon outside of tropical and sub-tropical regions [4]. Recently, increased travelling to and from endemic countries has resulted in the spread of dengue in new regions [2] [4] [5]. From 2010 to 2013, dengue outbreaks have been reported in several European countries and in the United States [4] [5]. The outbreaks are believed imported via travellers that visited endemic regions such as the Caribbean, Asia, and Latin America [4] [5]. Closer to home, Canadian authorities issued warning for travellers to be alert on dengue fever including what to do and to look for if they get infected [6].

Factors such as rapid population growth and the ease of migration also compound the problems of endemic nations. Close contact between human and vectors coupled with an increased size of the host population often increases the chances of contracting dengue [7]-[9]. The widespread occurrence of such population networks plays significant roles in distributing the disease, and pathologically, this condition often provide excellent room for disease to continue developing and growing [8] [10]. Additionally, global economic trade from endemic countries such as the shipment of used tires and changing weather patterns also contributes to the expansion of dengue infections beyond the vector's niche [7] [11] [12].

According to May 2015 update, the World Health Organisation (WHO) estimated that over 3.9 billion people worldwide are at risk of contracting dengue and there are roughly 284 - 528 million infections every year [13]. 500,000 people are hospitalised with severe dengue annually and $2.5 \%$ of them succumb to the infection [13]. Of all reported cases, most of the patients with severe infection of dengue are children [13]. With high numbers of infections and mortality rates, the dengue endemic can no longer be ignored.

As an example, dengue cases in Malaysia alone show an astonishing increment towards the end of 2014 with 43,346 cases reported from January to September 2013, while 76,700 cases were reported within the same window in 2014 [14]. Out of all these reported cases, another major increase of death toll was observed from 43 cases in 2013 to 146 cases in 2014 [14]. With an estimated increase of 76.9\% sufferers, dengue has become a major health issue in Malaysia, with local news media and the public criticizing their health ministry for failing to curb the increase in the prevalence of the disease [15]. According to Paul and Tham (2015), in January 2015, the numbers of infected individuals keep increasing by $59 \%$ when compared to similar statistic of the same month in 2014 [16].

Several combative measures across all endemic countries have been applied and tested to suppress the prevalence of dengue over the years and most common one is the heavy use of Aedes larvicide [7] [14]. Others include education on dengue pathogenesis and pathology for public and active extermination of mosquito breeding sites, which include all bodies of stagnant water [7] [14].

In this study, the use of vaccines and biological control will be discussed. Current research and experimental results of these dengue protective ideas will be discussed and evaluated in terms of their functionality, confidence, and application in endemic areas. In addition, problems that may arise upon the application of both measures once they are introduced indefinitely will be evaluated. The pathogenesis and pathology of dengue will also be discussed to provide strong arguments to support the use of vaccines and biological control in preventing dengue infection.

\section{Pathogenesis and Pathology of Dengue}

Transmitted by the mosquito vectors A. aegypti and A. albopictus, dengue is a viral type disease (arbovirus) and is categorised as viral hemorrhagic fevers (VHF) [1]-[4]. Common dengue serotypes called DENV (dengue virus) that are found globally are DENV-1, DENV-2, DENV-3, and DENV-4 [1]-[4] [7] [11] [13] [17]. Recently, dengue researchers have also found a new serotype of dengue, DENV-5, from infected patients in Borneo [18]. Out of this five serotypes, DENV-3 and DENV-4 have been associated with the Dengue Shock Syndrome (DSS) stage, whereby the infection deemed severe and often leads to fatalities [17]. As seen in Figure 1, DENV1 and DENV 3 are found to be highly associated with a 95\% species similarity, while DENV2 is closely related to a 


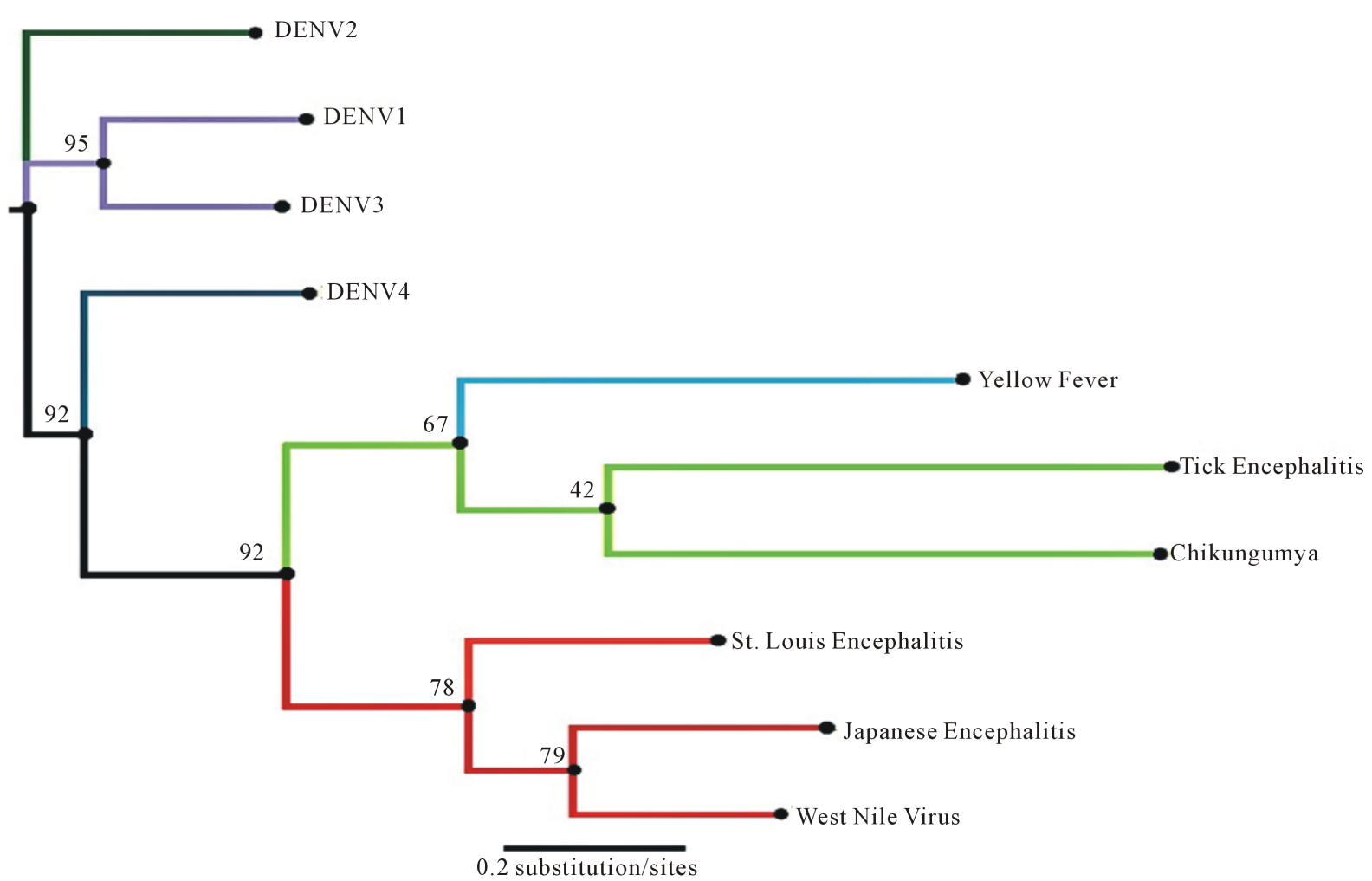

Figure 1. Phylogenetic Tree of Viral Hemorrhagic Fever (VHF) viruses of family Flaviviridae. Tree is made using complete genomic sequences obtained from National Center for Biotechnology Information (NCBI) of which accession numbers are given. Bootstrap values are illustrated between most nodes and based on calculation of 100 possible combinations. Higher bootstrap value signifies higher similarity between species of viruses. Phylogenetic illustration is made using the software Geneious and FigTree v1.4.2.

flaviviridae ancestor, and DENV4 is closely related to other flaviviridae viruses. The accession numbers of all serotypes used to construct the phylogenetic tree are M87512.1 (DENV1), M29095.1 (DENV2), AY662691.1 (DENV3), AF326825.1 (DENV4), NC_001672.1 (Tick-borne Encephalitis), AY 508813.1 (Japanese Encephalitis), NC_007580.2 (St. Louis Encephalitis), HM147824.1 (West Nile Virus), NC_ 004162.2 (chikungunya) and NC_002031.1 (Yellow Fever) [19]-[28].

Classified as a flavivirus, dengue is composed of a single positive-stranded RNA virus of the Flaviviridae family [4] [29]. Immunologically, viruses of RNA origin have proved to be difficult to treat due to their ability to rapidly change their genomic structures, or because pathogenicity islands may favour their development [18]. These factors have made these viruses highly successful in maintaining their reservoir and may become infectious in any environment or host they occupy. DENV is known to be equipped with three main protein structures and as many as seven non-structural proteins that encode for its pathogenicity [30]. These three main proteins; E-protein, prM/M protein, and C proteins are thought to be responsible for the component of the virus including attachment while the NS1, NS2A/B, NS3, NS4A/B, and NS5 non-structural proteins are deemed essential for its replication mechanism [30].

Jain et al. (2014) argue that DENV can infect a diverse range of insect or mammalian cells due to its structure [31]. This is typical for mosquito vector diseases in which a virus can 'hop' in from the arthropod host to an intermediate host [32]. When inoculated into humans, DENV will deliver itself to the liver cells (hepatocytes) and to the intestinal cells (enterocytes) for further development and replication [31] [33]. In severe infection, this virus can cause necrosis of these hepatocytes and enterocytes which often leads to tissues loss and blood loss [33].

Early detection of dengue infection can often save lives [3] [4]. Due to its latency within the first few days post infection, an infected person will not know he or she is infected until specific symptoms are expressed, as seen in Figure 2 [3] [4]. Normal symptoms of early infection are documented as headache, retro-orbital pain, muscle aches, joint pain, fever, and rashes [2]-[4]. These symptoms are often observed during the Dengue 


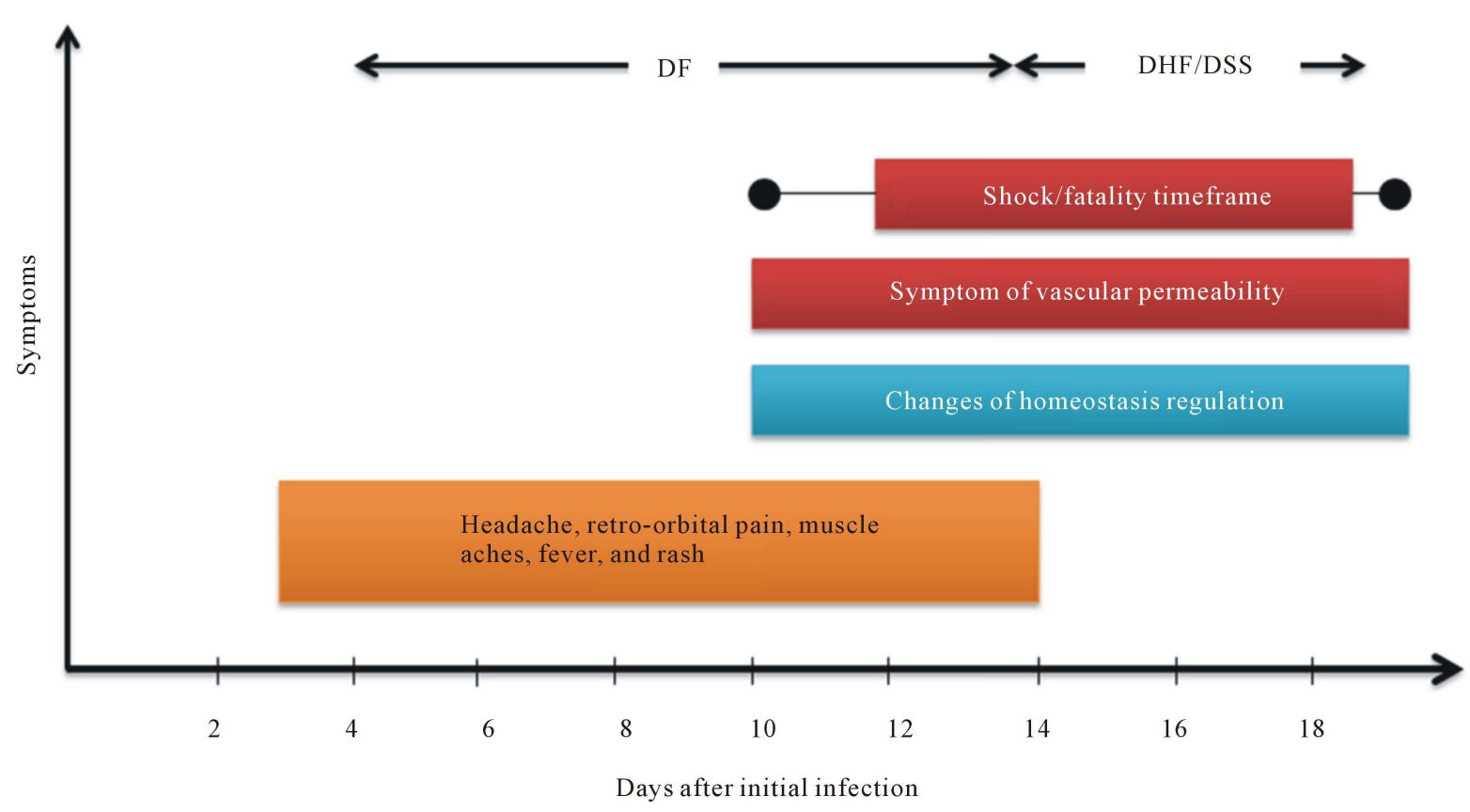

Figure 2. Adaptation of generalised timescale of dengue infection within a patient. Figure illustrates generalised symptoms of dengue including major infection stages. Symptoms according to collection literatures only exist after initial infection in a host. Figure is a summary of symptoms taken from various studies on dengue infections and pathologies.

Fever (DF) stage which is between 3 to 14 days post infection [2]-[4] [34]. About 10 days post infection, dengue will start to progress into a new phase called Dengue Haemorrhagic Fever (DHF) stage and is often coupled with the lethal DSS stage [2]-[4]. Within the DHF/DSS period, dengue infection can lead to shock, thus causing fatalities if left unattended and untreated [2]-[4]. In DSS stage, patients are often observed showing changes in the homeostatic regulation and vascular permeability coupled with appearance of bleeding out as their cell integrity is compromised and start to haemolyse [3] [9] [33] [35].

Although countless of researches had been conducted on dengue over the past 50 years of its discovery, the pathogenesis of dengue (specifically) is not yet entirely known and our understanding of this disease is limited [31] [36]. This will later influence our discussions on the choice of combative measures and their effectiveness when dealing with DENV.

\section{Dengue Vectors Biological Control}

The simplest concept of biological control is defined as a method to reduce pests using their natural antagonists to maintain equilibrium in a disrupted population [37] [38]. Dengue researchers have therefore developed the idea of using the Aedes mosquito's natural parasites or predators to supress the transmission of DENV and disrupt the life-cycle of the arthropod vector. These include utilising extensive studies of parasite-host interaction in Aedes vectors by the use of parasitic bacteria and more traditional method such as introducing predatory fishes into mosquito breeding sites [39]-[45]. The concept of introducing insect parasites as a means of suppressing dengue will be specifically discussed.

As humans, we harbour millions of other organisms in our body that live either as symbionts or parasites [46]. Similarly, insects of various classes and orders also have other organisms living freely within them. Specific parasites in dengue vectors can be manipulated to fight these vectors [41]-[45]. However, it is imperative that we look for the parasites that are present in all of the vector's life stages to increase the effectiveness and efficacy of biological control.

Marimuthu et al. (2013) discussed the idea of introducing "eco-friendly" bacteria parasites in mosquitoes because of the increasing resistance of mosquito larvae to commonly used larvicide. Bacillus thuringiensis was found to be highly specific and a common parasite in mosquitoes of the Diptera order, such as Aedes and Anopheles (Malaria) [39] [40]. The specificity is due to B. thuringiensis ability to produce a specific toxin ( $\delta$-toxin) 
that is toxic to mosquito larva, thus reducing the amount of emerging adults mosquitoes in the environment [39]. This however is no longer the case as recently there are reports indicating that mosquito's larva develop resistance against their bacterial parasites thus rendering useless the application of inoculating wild-type $B$. thuringiensis into larva population [39] [47]. However, if B. thuringiensis is inoculated with cobalt nanoparticles (Co-NPs), the effectiveness of bacteria toxicity is increased, thus reducing the resistance issues faced earlier [39]. Their results indicate that $100 \%$ of $A$. aegypti larva died after introduction of an 8 - 10 fold concentration of Co-NPs into the larval population whereas wild-type $B$. thuringiensis required a 20-fold concentration to achieve the same results [39]. Although with promising results, there are no indications that the result of the study is being applied at the moment. Other bacteria which had been studied as bacterial parasites for the mosquitoes include Bacillus cereus, Streptomyces sp., and Paecilomyces sp. [39].

Similar to $B$. thuringiensis, Wolbachia is also a species of insects' bacteria but is classified more as a symbiont rather than a parasite of mosquitoes [41]. Wolbachia is not species specific and it possesses symbiotic diversity towards other insects within the arthropod kingdom [41]. It is estimated that $25 \%$ to $70 \%$ of insects in the phylum Arthropoda are known to be infected with Wolbachia and become hosts [41]. Wolbachia targets adult mosquitoes instead of the larva and is found residing in the mid-gut and ovaries [42] [45]. Other locations of Wolbachia within the insect host include salivary glands and the brain, which may be correlated with reduced DENV transmission and replication [45]. The first observed pathology of Wolbachia in Aedes is that the bacteria induced restrictions on the reproduction process in infected female mosquitoes by changing their reproduction cycle and life-span, thus reducing their ability to produce progeny [48]. Wolbachia will modulate high iron level in the mosquito during blood meals, especially within the ovaries, thus disrupting the mosquito's reproduction cycle [45]. In addition, shorter life-spans are observed within affected populations, thus reducing the chances of female mosquito obtaining blood meals from their preferred host and to mate with male mosquito [42]. Other studies conducted also illustrate that infected female mosquito are less likely to produce offspring when mating with non-infected male mosquito and vice versa [43]. Results indicated mixed outcomes as one study illustrates that the prevalence of dengue transmission is discovered to be much lower if male Aedes is infected with Wolbachia while another study indicates lower dengue transmission with infected female Aedes [43] [44].

The parasite-host idea of using Wolbachia infected mosquitoes to control transmission of dengue was then tested in the field by releasing these mosquitoes into the environment to mate with the wild mosquito population. Field studies conducted in Australia show that the Wolbachia strain wMel managed to colonize most of the wild-type Aedes mosquitos' population and that the results correlate with significant reduction of DENV infection in a specific area [45]. In addition, the study also concluded that other RNA viruses transmitted by Aedes species such as malaria, chikungunya, yellow fever, and West Nile viruses are greatly reduced in Wolbachiainfected mosquito population [45]. Other similar studies had been conducted in other endemic areas globally and their results also show a significant reduction in DENV transmission by Wolbachia infected mosquitoes [15].

\section{Dengue Virus (DENV) Vaccine}

One of the popular choices in medicine to eradicate a disease is to use vaccines. Vaccines have demonstrated the ability to produce a major reduction in disease-related mortality and prevalence in a human population [49]. The first flavivirus vaccine successfully developed is Max Theiler’s YFD (yellow fever disease) vaccine in 1951 [50]. The vaccine was created using attenuated live virus in which has saved more than 500 million people globally since its introduction in 1937 [50].

From an immunological perspective, the usage of live-attenuated virus in vaccine is considered to be an effective way of inducing immune responses since attenuated virus can "teach" our immune system to readily recognize antigen molecular patterns upon subsequent infections [51]. The end product of this inoculation is the production of "licensed" memory cells during the humoral and adaptive immune stages, in which these cells will readily fight against further viral invasion [51].

The development of DENV vaccines should encompass all the serotypes present in order for it to be successful [2] [4] [18] [31] [36] [50]. A multivalent vaccine is important in dengue virus treatment as a vaccine that targets one serotype will only be specific to that serotype viral structures but not others [11] [36] [50] [52]. In an endemic area, a person can be infected with either one serotype or multiple serotypes of DENV [11] [36] [50] [52]. Targets of vaccines should encompass all the main protein structures as well as the non-structural proteins as discussed in the previous section. At the moment, available DENV vaccines produced or on production only 
target four out of five serotypes presence (mainly because DENV5 is new and a vaccine is yet to be developed) and is known as tetravalent vaccine [50] [52].

The idea of tetravalent vaccine, a concocted mixture containing all live-attenuated DENV serotypes to teach human immune response to recognize and target them, was first developed in the early 2000s at Mahidol University, Thailand as a novel way to combat the prevalence of DENV1-4 that affected many individuals in Southeast Asia [48]. Similarly as YFV vaccine, the dengue tetravalent vaccine is made by using live-attenuated virus of dengue of which serotypes DENV1, DENV2, and DENV4 attenuated viruses are developed using dog kidney cells and serotype DENV3 attenuated virus developed using African green monkey kidney cells [48] [53]. Attenuated viruses are subjected to bio-marker testing in the kidney cells of tested animals and follow procedures outline in Figure 3 prior to manufacturing a vaccine [53]. This early tetravalent vaccine was not deemed successful as the patient vaccinated with the vaccine showed occurrence of dengue-like symptoms after clinical testing [48]. The reason of the failure can be caused by different factors, and a plausible explanation is that the attenuated virus might regain its pathogenicity after inoculation. Further improvement is then made with the vaccine by other organizations in which it is renovated using a recombinant live-attenuated virus using mixture of live YFV virus with live dengue virus [48] [53]-[55]. The results differ significantly since the first clinical trial of a vaccinated patient showed immuno-competency on all four DENV serotypes and did not show any sign of dengue-like symptoms [48].

Thailand was the first country to test the redesigned tetravalent vaccine of TDEN (Tetravalent Dengue) that targeted all four serotypes, and clinical results indicate symptoms and clinical signs of dengue were greatly reduced to mild, or moderate, and or transient stages [52]. Additionally, the team of researchers had also given promising review that the TDEN vaccine does not result in serious vaccines-related side-effects [52]. In Malaysia, another tetravalent vaccine called CYD-TDV (tetravalent candidate dengue vaccine) is utilised [56]. The target comprises of healthy children aged 2 - 11 years and clinical results are satisfactory, whereby the humoral immune system, responsible for antigen-viral recognition, respond to all four DENV serotypes [56]. Similar studies in Thailand and their clinical results indicate that the CYD-TDV vaccine protected those vaccinated against all four serotypes and volunteers did not express side-effects after vaccination [56].

Recently, Sanofi-Pasteur announced that they have managed to develop a successful dengue vaccine in which their final clinical trial shows a tremendous reduction of $60.8 \%$ of dengue infection in vaccinated volunteers [57] [58]. These volunteers comprised of more than 20,000 children aged 9 - 16 from countries in Latin America, and were observed to be highly protected against dengue infection, specifically in the DHF stages [57]. In addition, it is estimated that roughly around $80 \%$ of these volunteers escaped the risk of hospitalisation and over $90 \%$ are protected against severe dengue symptoms [58]. The vaccine used for this clinical trial is of CYD-TDV similar to that utilised in clinical trials in Thailand and Malaysia. The method of administering the vaccine is done in three-dose vaccinations over 25 months [58]. Although promising, the published study involved only volunteers from one geographical region. However, Sanofi-Pasteur's head of research and development assured the public

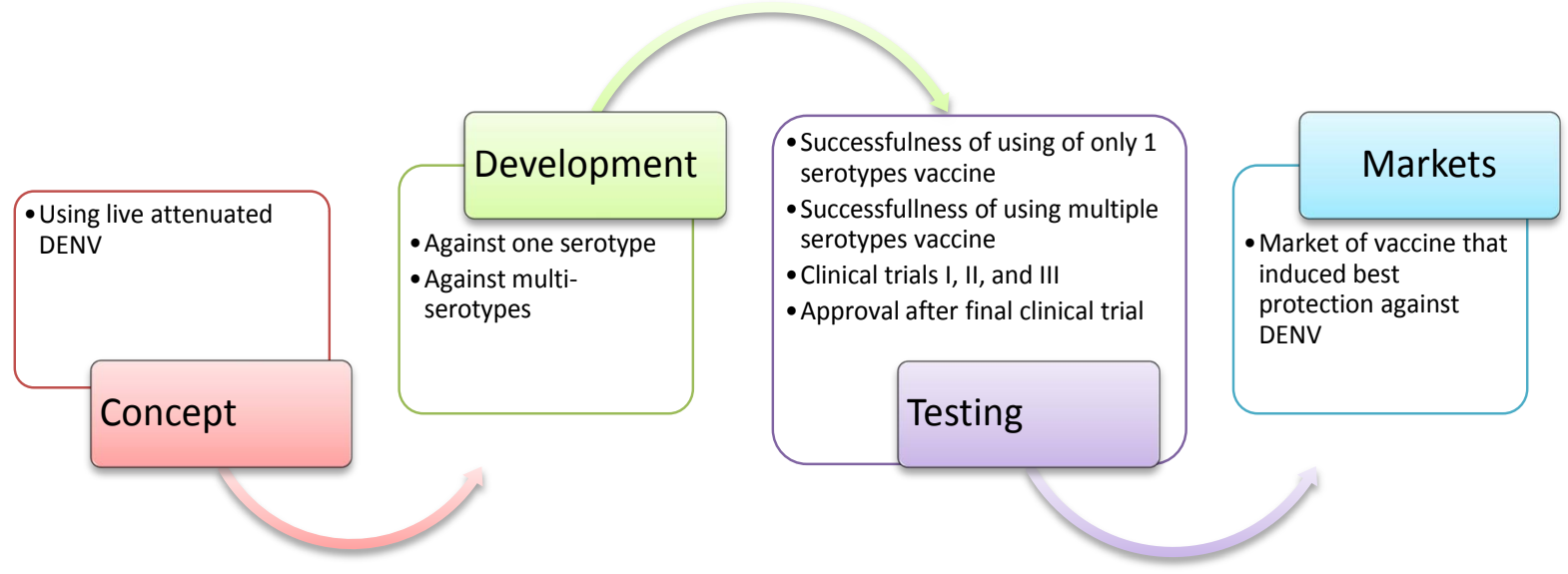

Figure 3. Step in utilising concept to develop effective vaccine. Model illustrates the simplified pathway in creating effective vaccines before being introduced permanently to publics. 
that the vaccine could work effectively given the outstanding and consistent results [58]. Overall, Sanofi's clinical trials had involved more than 40,000 volunteers across the globe and these volunteers are predominantly those that live in endemic areas of Asia and Latin America [59].

\section{Discussion}

Both biological control and vaccination present outstanding results in managing the prevalence of dengue in their experimental areas. We are now left with a choice between the aforementioned combative measures in order to assure maximum effectiveness and towards reducing dengue.

Biological control enables the manipulation of the parasite-host interaction or symbiont to reduce dengue transmission in endemic areas. Two studies that we looked into were the use of parasitic bacteria B. thuringiensis and symbiotic bacteria Wolbachia. Although both produced outstanding results, we encountered several problems related to the manipulation of the relationship between these bacteria and Aedes mosquitoes. First, the mosquitoes' populations inoculated with $B$. thuringiensis are shown to develop resistance towards $B$. thuringiensis after period of time, signifying that the Aedes mosquito might possess immunity against its parasitic tenants [39] [47]. Secondly, mosquitos inoculated with Wolbachia shows preference in shutting down transmission of certain serotype of DENV rather than all serotypes. Frentiu et al. (2014) illustrate that Wolbachia-infected mosquitoes shut down the transmission of DENV1 but continued transmitting DENV2 and DENV 3 [45]. It is mentioned that the DENV3 serotype is one of the two severe serotypes of DENV that may induce shock and death. Aedes may also develop resistance towards Wolbachia symbiont as illustrated by the case of $B$. thuringiensis. Thus, studies concerning mosquito immunology are important in preventing the possibility of mosquito developing resistance. Other concerns are the environmental impact on the overall ecosystem. In Malaysia for example, environmentalists are against the introduction of Wolbachia infected mosquitoes into the wild for reason that it may pollute Malaysia's pristine rainforest condition [15]. However several studies conducted on usage of treated mosquito assured the public that introduction of these mosquitoes does not risk any potential damage to the environment and the existing food chain.

In vaccination, the main problem that may arise is the issue of resistance towards the vaccine itself. Viruses are known to have rapid replication and can interchange genomes depending on whether the viruses benefit from the changes or not. This creates a situation in which the development of new vaccines tends to become obsolete due to the fast changes in viruses' genomics structures. This inevitable issue creates another problem in which pharmaceutical companies completely stop producing new vaccine as they do not see the incentives in continuing as it is deemed economically unprofitable [60]. Next is the issue of using live-attenuated viruses in vaccine. Although attenuated, small percentage of these viruses may revert back to their original pathology, thus resulting in accidental secondary DENV infection towards those vaccinated [52] [61]. As shown in the study of first tetravalent vaccine, vaccinated patients show a recurrence in dengue-like infection post vaccination [48]. Method of delivery and stability of the vaccine might also pose an issue for a vaccine to effectively work. In major endemic areas, most of the vulnerable individuals live beyond reach of modernization and this creates the problem of accessing electricity needed to cool live attenuate virus in order to keeping them stable [61]. In addition, an effective dengue vaccine needs to encompass all serotypes in existence for optimal effectiveness. Although Sanofi-Pasteur successfully created the functional tetravalent dengue vaccine, they would need to factor in the emergence DENV5 in Southeast Asia. Another possible problem with vaccine is that it cannot be used to treat immuno-competent patient as it may lead to other secondary infections in that patient [60]. The suggestions to improve the efficacy of vaccine are to develop new vaccines to target specific sites in DENV that cannot be interchanged, creating stabilised vaccines that can be delivered at any temperature without the chances of reversion, and continual research and study of drugs efficient enough against the virus but do not adversely affect the intended host [61].

Overall, biological control approach seems to be a viable option at the moment as it deals with the issue of transmission at its root and is inexpensive to cultivate [42]-[45]. Although it may be slightly problematic, the approach tends to be an excellent and effective combative measure in long-term protection against dengue transmission in any endemic areas. Note that for added protection, vaccination should also be considered as this approach can help induce a stronger immune response against dengue infection. Education about dengue's pathology could also help to reduce dengue transmission, since everyone will feel responsible for maintaining the cleanliness of his or her environment [3]. However, as mentioned previously, we still do not know much about 
this disease and a lot is still needed to be done to understand it. Therefore, more research is still needed on these two combative measures to make them impenetrable to dengue replication and reproduction. Suggestion of researches in the future are to create novel vaccine that directly target main proteins that are essential for DENV attachment regardless of serotypes whilst weakening its non-structural proteins and finding a sustainable ecological approach to weaken DENV transmission via introduction of a novel parasite-symbiont bacteria that is counter-resistant to mosquitos and larvae capabilities of manipulating their genome to resist bacterial infections.

\section{Acknowledgements}

Writing is conducted by B.P and T.W.L. Editing and proofreading is conducted by W.L.T. Research, data collections, and analysis is conducted by B.P. Data and statistic on Malaysia's Dengue can be obtained from World Health Organisation (WHO) at http://www.who.int/mediacentre/factsheets/fs117/en/. Article is produced by undergraduates, B.P (UAlberta) and T.W.L. (UBC).

\section{References}

[1] (2014) Mosquito-Borne Diseases. American Mosquito Control Association. http://www.mosquito.org/mosquito-borne-diseases

[2] McFee, R.B. (2013) Viral Hemorrhagic Fever Viruses. Disease-a-Month, 59, 410-425. http://dx.doi.org/10.1016/j.disamonth.2013.10.003

[3] (2014) Sign and Symptom of Dengue Virus. http://www.denguevirusnet.com/signs-a-symptoms.html

[4] Mangold, K. and Reynolds, S. (2013) Review of Dengue Fever. Pediatric Emergency Care, 29, 665-669. http://dx.doi.org/10.1097/PEC.0b013e31828ed30e

[5] Wilson, M.E., Weld, L.H., Boggild, A., Keystone, J.S., Kain, K.C., Sonnenburg, F. and Schwartz, E. (2007) Fever in Return Travellers: Result from the Geosentinel Surveillance Network. Clinical Infectious Diseases, 44, 1560-1568. http://dx.doi.org/10.1086/518173

[6] Government of Canada (2014) Dengue Fever. http://travel.gc.ca/travelling/health-safety/diseases/dengue

[7] Sizmur, C. (2014) Malaysia Steps up Campaign against Dengue. http://www.publichealth.basf.com/agr/ms/public-health/en GB/content/public-health/our-partners/Dengue/dengue in malaysia

[8] Hu, H., Nigmatulina, K. and Eckhoff, P. (2013) The Scaling of Contact Rates with Population Density for the Infectious Disease Models. Mathematical Biosciences, 244, 125-134. http://dx.doi.org/10.1016/j.mbs.2013.04.013

[9] Tam, J.S., Barbeschi, M., Shapovalova, N., Briand, S., Memish, Z.A. and Kieny, M.P. (2012) Research Agenda for Mass Gatherings: A Call to Action. Lancet Infection, 12, 231-239. http://dx.doi.org/10.1016/s1473-3099(11)70353-x

[10] Miller, J.C. and Voltz, E.M. (2013) Incorporating Disease and Population Structure into Models of SIR Disease in Contact Networks. PloS One, 8, 1-14. http://dx.doi.org/10.1371/journal.pone.0069162

[11] Wongkoon, S., Jaroensutasinee, M. and Jaroensutasinee, K. (2013) Distribution, Seasonal Variation \& Dengue Transmission Prediction in Sisaket, Thailand. Indian Journal of Medical Research, 138, 347-353.

[12] Colon-Gonzalez, F.J., Fezzi, C., Lake, I.R. and Hunter, P.R. (2013) The Effects of Weather and Climate Change on Dengue. PLoS Neglected Tropical Diseases, 7, 1-9. http://dx.doi.org/10.1371/journal.pntd.0002503

[13] WHO (2015) Dengue Fever Fact Sheet No 117. Updated January 2015. http://www.who.int/mediacentre/factsheets/fs117/en/

[14] Malaysia Remote Sensing Agency (2014) http://idengue.remotesensing.gov.my/.

[15] Sue-Chern, L. (2014) As Dengue Death Rise, Will Malaysia Try Breeding Out "Bad” Aedes? The Malaysian Insider. http://www.themalaysianinsider.com/malaysia/article/as-dengue-deaths-rise-will-malaysia-try-breeding-out-bad-aedes

[16] Paul, B. and Tham, W.L. (2015) Interrelation between Climate and Dengue in Malaysia. Health, 7, 672-678. http://dx.doi.org/10.4236/health.2015.76080

[17] Yacouba, S., Mongkolsapayaa, J. and Screatona, G. (2013) Pathogenesis of Dengue. Current Opinion of Infectious Disease, 26, 284-289. http://dx.doi.org/10.1097/QCO.0b013e32835fb938

[18] Normile, D. (2014) Surprising New Dengue Virus Throws a Spanner in Disease Control Efforts. Science, 342, 415. http://dx.doi.org/10.1126/science.342.6157.415

[19] Fu, J., Tan, B.H., Yap, E.H., Chan, Y.C. and Tan, Y.H. (1992) Full-Length cDNA Sequence of Dengue Type 1 Virus (Singapore Strain S275/90). Virology, 188, 953-958. http://dx.doi.org/10.1016/0042-6822(92)90560-C 
[20] Putnak, J.R., Charles, P.C., Padmanabhan, R., Irie, K., Hoke, C.H. and Burke, D.S. (1988) Functional and Antigenic Domains of the Dengue-2 Virus Nonstructural Glycoprotein NS-1. Virology, 163, 93-103. http://dx.doi.org/10.1016/0042-6822(88)90236-X

[21] Lim, S.P., Ooi, E.E. and Vasudevan, S.G. (2004) Full Length Genomic Sequence of a Dengue Virus of Serotype 3 from Singapore. (Unpublished)

[22] Durbin, A.P., Karron, R.A., Sun, W., Vaughn, D.W., Reynolds, M.J., Perreault, J.R., Thumar, B., Men, R., Lai, C.J., Elkins, W.R., Chanock, R.M., Murphy, B.R. and Whitehead, S.S. (2001) Attenuation and Immunogenicity in Humans of a Live Dengue Virus Type-4 Vaccine Candidate with a 30 Nucleotide Deletion in Its 3'-Untranslated Region. Annual Journal of Tropical Medicine Hygiene, 65, 405-413.

[23] Wallner, G., Mandl, C.W., Kunz, C. and Heinz, F.X. (1995) The Flavivirus 3'-Noncoding Region: Extensive Size Heterogeneity Independent of Evolutionary Relationships among Strains of Tick-Borne Encephalitis Virus. Virology, 213, 196-178. http://dx.doi.org/10.1006/viro.1995.1557

[24] Shah, P.S., Tanaka, M., Khan, A.H., Mathenge, E.G., Fuke, I., Takagi, M., Igarashi, A. and Morita, K. (2006) Molecular Characterization of Attenuated Japanese Encephalitis Live Vaccine Strain ML-17. Vaccine, 24, 402-411. http://dx.doi.org/10.1016/j.vaccine.2005.10.048

[25] Ciota, A.T., Lovelace, A.O., Ngo, K.A., Le, A.N., Maffei, J.G., Franke, M.A., Payne, A.F., Jones, S.A., Kauffman, E.B. and Kramer, L.D. (2007) Cell-Specific Adaptation of Two Flaviviruses Following Serial Passage in Mosquito Cell Culture. Virology, 357, 165-174. http://dx.doi.org/10.1016/j.virol.2006.08.005

[26] McMullen, A.R., Albayrak, H., May, F.J., Davis, C.T., Beasley, D.W. and Barrett, A.D. (2013) Molecular Evolution of Lineage 2 West Nile Virus. Journal of General Virology, 94, 318-325. http://dx.doi.org/10.1099/vir.0.046888-0

[27] Rice, C.M., Lenches, E.M., Eddy, S.R., Shin, S.J., Sheets, R.L. and Strauss, J.H. (1985) Nucleotide Sequence of Yellow Fever Virus: Implications for Flavivirus Gene Expression and Evolution. Science, 229, 726-733. http://dx.doi.org/10.1126/science.4023707

[28] Khan, A.H., Morita, K., Parquet, M., Parquet, M. del C., Mathenge, E.G. and Igarashi, A. (2002) Complete Nucleotide Sequence of Chikungunya Virus and Evidence for an Internal Polyadenylation Site. Journal of General Virology, 83, 3075-3084. http://dx.doi.org/10.1099/0022-1317-83-12-3075

[29] Rodenhuis-Zybert, I.A., Wilschut, J. and Smit, J.M. (2010) Dengue Virus Life Cycle: Viral and Host Factors Modulating Infectivity. Cellular and Molecular Life Sciences, 67, 2773-2786. http://dx.doi.org/10.1007/s00018-010-0357-z

[30] Perera, R. and Kuhn, R.J. (2008) Structural Proteomics of Dengue Virus. Current Opinion in Microbiology, 11, 169178. http://dx.doi.org/10.1016/j.mib.2008.06.004

[31] Jain, B., Chaturvedi, U.C. and Jain, A. (2014) Role of Intracellular Events in the Pathogenesis of Dengue: An Overview. Microbial Pathogenesis, 69-70, 45-48. http://dx.doi.org/10.1016/j.micpath.2014.03.004

[32] Belosovic, M. (2014) Plasmodium sp. Zoology 352 Lecture Series, University of Alberta, Edmonton.

[33] Buret, A.G. (2014) Pathogenesis of Parasitic Disease. Zoology 352 Lecture Series, University of Alberta, Edmonton.

[34] Gubler, D.J. (2010) Dengue Viruses. In: Mahy, B.W.J. and Van Regenmortel, M.H.V., Eds., Desk Encyclopedia of Human and Medical Virology, Academic Press, Boston, 372-382.

[35] Povoa, T.F., Alves, A.M.B., Oliveira, C.A.B., Nuovo, G.J., Chagas, V.L.A. and Paes, M.V. (2014) The Pathology of Severe Dengue in Multiple Organs of Human Fatal Cases: Histopathology, Ultrastructure and Virus Replication. PloS ONE, 9, 1-16. http://dx.doi.org/10.1371/journal.pone.0083386

[36] Ishikawaa, T., Yamanakab, A. and Konishi, E. (2014) A Review of Successful Flavivirus Vaccines and the Problems with Those Flaviviruses for Which Vaccines Are Not Yet Available. Vaccine, 32, 1326-1337. http://dx.doi.org/10.1016/j.vaccine.2014.01.040

[37] Hoffmann, M.P. and Frodsham, A.C. (1993) Natural Enemies of Vegetable Insect Pests. Cooperative Extension, Cornell University, Ithica, 63 p.

[38] Bellows, T.S. (2001) Restoring Population Balance through Natural Enemy Introductions. Biological Control, 21, 199205. http://dx.doi.org/10.1006/bcon.2001.0936

[39] Marimuthu, S., Rahuman, A.A., Kirthi, A.V., Santhoshkumar, T., Jayaseelan, C. and Rajakumar, G. (2013) EcoFriendly Microbial Route to Synthesize Cobalt Nanoparticles Using Bacillus thuringiensis against Malaria and Dengue Vectors. Parasitology Resources, 112, 4105-4112. http://dx.doi.org/10.1007/s00436-013-3601-2

[40] Lee, H.L., Chen, C.D., Masri, S.M., Chiang, Y.F., Chooi, K.H. and Benjamin, S. (2008) Impact of Larvaciding with a Bacillus thuringiensis isrealensis Formulation, VectoBac WG, on Dengue Mosquito Vectors in a Dengue Endemic Site in Selangor State Malaysia. Southeast Asian Journal Tropical Medicine Public Health, 39, 601-609.

[41] Kozek, W. and Rao, R. (2007) The Discovery of Wolbachia in Arthropods and Nematodes-A Historical Perspective. Issues in Infectious Diseases, 5, 1-14. http://dx.doi.org/10.1159/000104228 
[42] Turley, A.P., Moreira, L.A., O’Neill, S.L. and McGraw, E.A. (2009) Wolbachia Infection Reduces Blood-Feeding Success in the Dengue Fever Mosquito, Aedesaegypti. PLoS Neglected Tropical Diseases, 3, 1-7. http://dx.doi.org/10.1371/journal.pntd.0000516

[43] Hancock, P.A., Sinkins, S.P. and Godfray, H.C.J. (2011) Strategies for Introducing Wolbachia to Reduce Transmission of Mosquito-Borne Diseases. PLoS Neglected Tropical Diseases, 5, 1-10. http://dx.doi.org/10.1371/journal.pntd.0001024

[44] Hoffmann, A.A., Iturbe-Ormaetxe, I., Callahan, A.G., Phillips, B.L., Billington, K., Axford, J.K., Montgomery, B., Turley, A.P. and O’Neill, S.L. (2014) Stability of the wMel Wolbachia Infection Following Invasion into Aedesaegypti Populations. PLoS Neglected Tropical Diseases, 8, 1-9. http://dx.doi.org/10.1371/journal.pntd.0003115

[45] Frentiu, F.D., Zakir, T., Walker, T., Popovici, J., Pyke, A.T., Van Der Hurk, A., McGraw, A.E. and O’Neill, S.L. (2014) Limited Dengue Virus Replication in Field-Collected Aedesaegypti Mosquitoes Infected with Wolbachia. PLoS Neglected Tropical Disease, 8, e2688. http://dx.doi.org/10.1371/journal.pntd.0002688

[46] Baron, S., Ed. (1996) Medical Microbiology. 4th Edition, The University of Texas Medical Branch at Galveston, Galveston.

[47] Chatterjee, S., Ghosh, T.S. and Das, S. (2010) Virulence of Bacillus cereus as Natural Facultative Pathogen of Anopheles subpictus Grassi (Diptera: Culicidae) Larvae in Submerged Rice-Fields and Shallow Ponds. African Journal of Biotechnology, 9, 6983-6986.

[48] Dayan, G.H., Thakur, M., Boaz, M. and Johnson, C. (2013) Safety and Immunogenicity of Three Tetravalent Dengue Vaccine Formulations in Healthy Adults in the USA. Vaccine, 31, 5048-5055. http://dx.doi.org/10.1016/j.vaccine.2013.08.088

[49] McNeil, M.M., Gee, J., Weintraub, E.S., Belongia, E.A., Lee, G.M., Glanz, J.M., Nordin, J.D., Klein, N.P., Baxter, R., Naleway, A.L., Jackson, L.A., Omer, S.B., Jacobsen, S.J. and DeStefano, F. (2014) The Vaccine Safety Datalink: Successes and Challenges Monitoring Vaccine Safety. Vaccine, 32, 5390-5399. http://dx.doi.org/10.1016/j.vaccine.2014.07.073

[50] Heinz, F.X. and Stiasny, K. (2012) Flaviviruses and Flavivirus Vaccines. Vaccine, 30, 4301-4307. http://dx.doi.org/10.1016/j.vaccine.2011.09.114

[51] Hemming, D. (2014) Immune Regulation to Post 2014. Immunology 371 Lecture Series, University of Alberta, Edmonton.

[52] Watanaveeradej, V., Gibbons, R.V., Simasathien, S., Nisalak, A., Jarman, R.G., Kerdpanich, A., Tournay, E., De La Barrerra, R., Dessy, F., Toussaint, J.F., Eckels, K.H., Thomas, S.J. and Innis, B.L. (2014) Safety and Immunogenicity of a Rederived, Live-Attenuated Dengue Virus Vaccine in Healthy Adults Living in Thailand: A Randomized Trial. American Journal of Tropical Medicine and Hygiene, 91, 119-128. http://dx.doi.org/10.4269/ajtmh.13-0452

[53] Bhamarapravati, N. and Sutee, Y. (2000) Live Attenuated Tetravalent Dengue Vaccine. Vaccine, 18, 44-47. http://dx.doi.org/10.1016/S0264-410X(00)00040-2

[54] Guirakhoo, F., Weltzin, R., Chambers, T.J., Zhang, Z.-X., Soike, K., Ratterree, M., Arroyo, J., Georgakopoulos, K., Catalan, J. and Monath, T.P. (2000) Recombinant Chimeric Yellow Fever-Dengue Type 2 Virus Is Immunogenic and Protective in Nonhuman Primates. Journal of Virology, 74, 5477-5485. http://dx.doi.org/10.1128/JVI.74.12.5477-5485.2000

[55] Guirakhoo, F., Kitchener, S., Morrison, D., Forrat, R., McCarthy, K., Nichols, R., Yoksan, S., Duan, X., Ermak, T.H., Kanesa-Thasan, N., Bedford, P., Lang, J., Quentin-Miller, M.J. and Monath, T.P. (2006) Live Attenuated Chimeric Yellow Fever Dengue Type 2 (ChimeriVax-DEN2) Vaccine: Phase I Clinical Trial for Safety and Immunogenicity: Effect of Yellow Fever Pre-Immunity in Induction of Cross Neutralizing Antibody Responses to All 4 Dengue Serotypes. Human Vaccine, 2, 60-67. http://dx.doi.org/10.4161/hv.2.2.2555

[56] Hss, A.-S., Koh, A.-T., Tan, K.K., Chan, L.G., Zhou, L., Bouckenooghe, A., Crevat, D. and Hutagalung, Y. (2013) Safety and Immunogenicity of a Tetravalent Dengue Vaccine in Healthy Children Aged 2-11 Years in Malaysia: A Randomized, Placebo-Controlled, Phase III Study. Vaccine, 31, 5814-5822. http://dx.doi.org/10.1016/j.vaccine.2013.10.013

[57] Huet, N. (2014) Final Trial Confirms Efficacy of Sanofi’s Dengue Vaccine. Reuters. http://www.reuters.com/article/2014/09/03/us-sanofi-dengue-idUSKBN0GY0C520140903

[58] Villar, L., Dayan, G.H., Arredondo-García, J.L., Rivera, D.M., Cunha, R., Deseda, C., Reynales, H., Costa, M.S., Morales-Ramírez, J.O., Carrasquilla, G., Rey, L.C., Dietze, R., Luz, K., Rivas, E., Montoya, M.C.M., Supelano, M.C., Zambrano, B., Langevin, E., Boaz, M., Tornieporth, N., Saville, M. and Noriega, F. (2015) Efficacy of a Tetravalent Dengue Vaccine in Children in Latin America. The New England Journal of Medicine, 372, 113-123. http://dx.doi.org/10.1056/NEJMoa1411037

[59] Bernal, A. (2014) The World’s First, Large-Scale Dengue Vaccine Efficacy Study Successfully Achieved Its Primary 
Endpoint. Sanofi Pasteur Media.

http://www.sanofipasteur.com/en/articles/theworld-s-first-large-scale-dengue-vaccine-efficacy-study-successfully-achi eved-its-primary-clinical-endpoint.aspx

[60] Belosevic, M. (2014) Concept of Integrated Control against Parasites. Zoology 352 Lecture Series, University of Alberta, Edmonton.

[61] Buret, A. (2014) Drugs and Parasites. Zoology 352 Lecture Series, University of Alberta, Edmonton. 